Tema: Solidificação / Lingotamento

\title{
SIMULAÇÃO COMPUTACIONAL FLUIDODINÂMICA DO ESCOAMENTO E DA SOLIDIFICAÇÃO NO LINGOTAMENTO CONTÍNUO DE MOLDES REDONDOS*
}

\author{
Rodrigo Camargos Barroso ${ }^{1}$ \\ Rubens de Paula Machado ${ }^{2}$ \\ Matias Correa de Miranda ${ }^{3}$ \\ José Ernesto de Azevedo Nadalon \\ André Carlos Contini ${ }^{5}$ \\ Leonardo Barboza Trindade
}

\section{Resumo}

Os fenômenos de solidificação no lingotamento contínuo são altamente dependentes da velocidade de lingotamento e das condições de extração de calor do molde. Por sua vez, equipamentos como os agitadores eletromagnéticos, alteram significativamente o escoamento na região do molde, influenciando o perfil térmico e ajudando na redução do calor de superheat. Simular simultaneamente o escoamento, o transporte de energia e a solidificação é um dos objetivos deste trabalho. O modelo emprega o software Ansys/Fluent e é aplicado ao lingotamento contínuo de moldes redondos. Como resultado, obteve-se os mapas de temperatura e a espessura de casca na saída do molde para diferentes velocidades de lingotamento e correntes de agitação. Experimentos realizados em planta buscam comprovar as previsões do modelo. Espera-se, por fim, criar um padrão operacional no lingotamento contínuo buscando otimizar o uso do agitador eletromagnético em função da velocidade de lingotamento e da temperatura de superheat.

Palavras-chaves: Solidificação; Lingotamento contínuo; Agitação eletromagnética; Simulação computacional de fluidos (CFD).

\section{SIMULATION OF FLUID FLOW AND SOLIDIFICATION IN CONTINUOUS CASTING ROUND BILLETS}

\section{Abstract}

The solidification phenomena during the continuous casting are highly dependent on the casting speed and the heat extraction in mold. At the same time, devices as the electromagnetic stirrers change significantly the flow in the mold, affecting the thermal profile and helping to reduce the superheat. The aim of this work is to simulate simultaneously the flow, the energy transport and the solidification. The model employs the Ansys/Fluent package and it is applied to round billet continuous casting. As results, the temperature maps and the thickness of the solidified shell at the mold exit were obtained for different casting speeds and stirrer currents. Some tests carried out in the steel plant aims to confirm the model predictions. It is expected, lastly, to create an operation pattern in the continuous casting optimizing the electromagnetic stirring in function of the casting speed and the superheat temperature.

Keywords: Solidification; Continuous casting; Electromagnetic stirring; Computational fluid dynamics (CFD).

1 Engenheiro Metalurgista, MsC, Superintendência de Siderurgia, Vallourec Tubos do Brasil, Belo Horizonte, MG, Brasil.

2 Tecnólogo de Normalização e Qualidade, Gerência de Aciaria, Vallourec Tubos do Brasil, Belo Horizonte, MG, Brasil.

3 Técnico Industrial, Gerência de Qualidade e Pesquisa Siderúrgica, Vallourec Tubos do Brasil, Belo Horizonte, MG, Brasil.

4 Engenheiro Civil, MsC em Engenharia Metalúrgica, Pesquisador, STE, Porto Alegre, RS, Brasil.

5 Físico, MsC em Engenharia Metalúrgica, Pesquisador, STE, Porto Alegre, RS, Brasil.

6 Físico, Doutor em Engenharia Metalúrgica, Diretor, STE, Porto Alegre, RS, Brasil.

* Contribuição técnica ao $45^{\circ}$ Seminário de Aciaria - Internacional, 25 a 28 de maio de 2014, 


\section{INTRODUÇÃO}

A Vallourec Tubos do Brasil busca, através de projetos de Pesquisa e Desenvolvimento, a melhoria contínua da qualidade de barras lingotadas com vistas à redução de defeitos nos tubos e à otimização do próprio processo de lingotamento. Em 2013, no Seminário de Aciaria de 2013, Barroso et al. [1] mostrou que defeitos internos nos tubos podem estar associados, dentre outras coisas, com segregação e/ou porosidade central nas barras de aço. Um dos recursos disponíveis no lingotamento contínuo que podem contribuir para a melhoria da qualidade interna da barra é o agitador eletromagnético no molde. De acordo com Kunstreich [2], a agitação eletromagnética promove homogeneidade térmica na zona do molde, aumentando com isso a zona equiaxial de solidificação, mesmo para temperatures de superheat mais elevadas, conforme ilustrado pela Figura 1. Com isso, é esperada uma redução no aparecimento de segregação e, por consequência, uma queda na ocorrência de defeitos internos nos tubos.

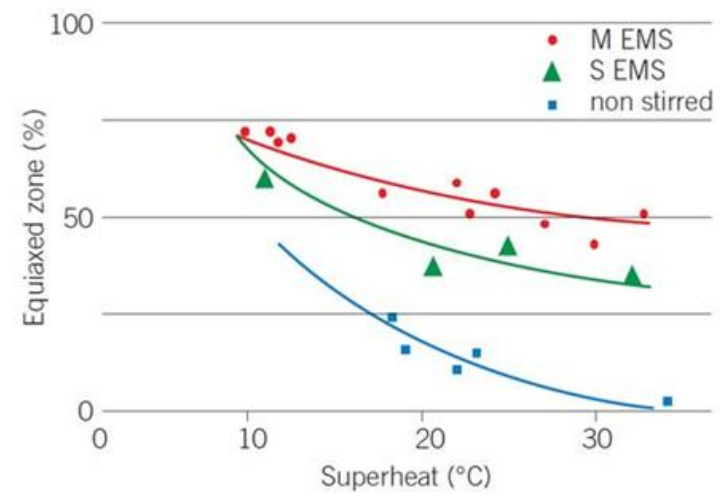

Figura 1. Largura da zona equiaxial em função do superheat sem e com agitador eletromagnético [2].

Barroso et al. [1] então avaliou a remoção do superheat através de simulação computacional do escoamento, com e sem agitação eletromagnética. Os resultados indicaram que a agitação eletromagnética no molde auxilia na extração de calor, reduzindo os efeitos do calor de superheat. Ao mesmo tempo, a agitação eletromagnética produz um perfil de temperatura mais homogêneo do aço líquido. Por fim, observou-se, experimentalmente, que o aumento da corrente de agitação foi capaz de promover um aumento da zona equiaxial de solidificação.

Porém, na ocasião, o modelo utilizado não considerava a solidificação. O desafio então, que é o objetivo deste trabalho, foi simular os efeitos de solidificação no modelo fluidodinâmico. Parâmetros como espessura de casca solidificada e mapas de fração líquida/sólida puderam ser obtidos sob diferentes condições operacionais de agitação eletromagnética no molde, velocidades e temperatura de lingotamento.

A literatura apresenta alguns trabalhos envolvendo a simulação simultânea de fenômenos de escoamento e solidificação no lingotamento contínuo de aços. Um dos pioneiros deve ter sido o trabalho realizado por Aboutalebi, Hasan e Guthrie [3], na qual os autores desenvolveram um modelo acoplado de escoamento, transferência de calor, solidificação e transporte de massa para simular a segregação de carbono em uma máquina de lingotamento de tarugos quadrados e redondos. Resultados de espessura de casca foram comparados com experimentos em planta, como mostra a Figura 2.

* Contribuição técnica ao 45 Seminário de Aciaria - Internacional, 25 a 28 de maio de 2014, 

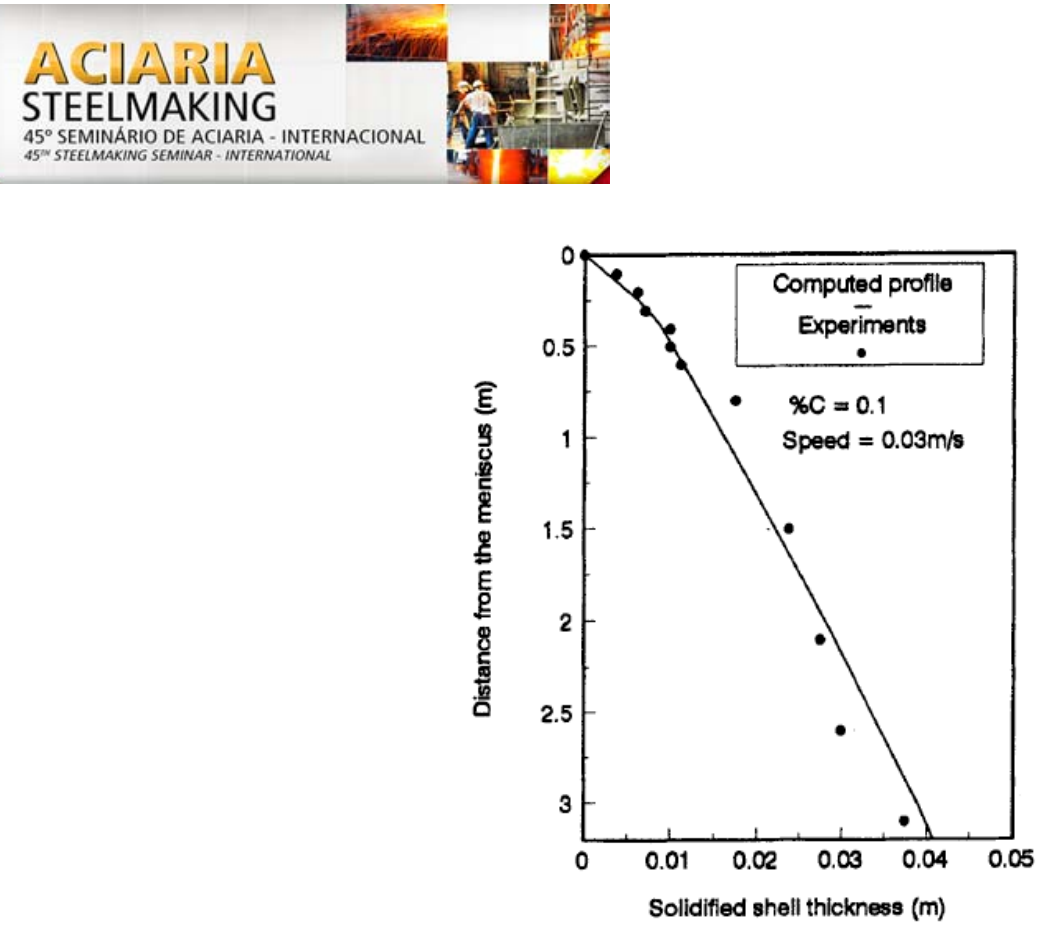

Figura 2. Comparação entre a espessura de casca calculada pelo modelo com os experimentos realizados por Ushijima. Fonte: Aboutalebi, Hasan e Guthrie [3].

Seyedein e Hasan [4] também desenvolveram um modelo 3D para a solução acoplada de escoamento, transferência de calor e solidificação, porém neste caso foi aplicado ao lingotamento contínuo de placas. Ha e colaboradores [5] deram continuidade ao trabalho de Seyedein e Hasan [4] incluindo o efeito de freios eletromagnéticos, tradicionalmente empregados em placas, na solidificação. Alguns resultados estão dispostos na Figura 3.

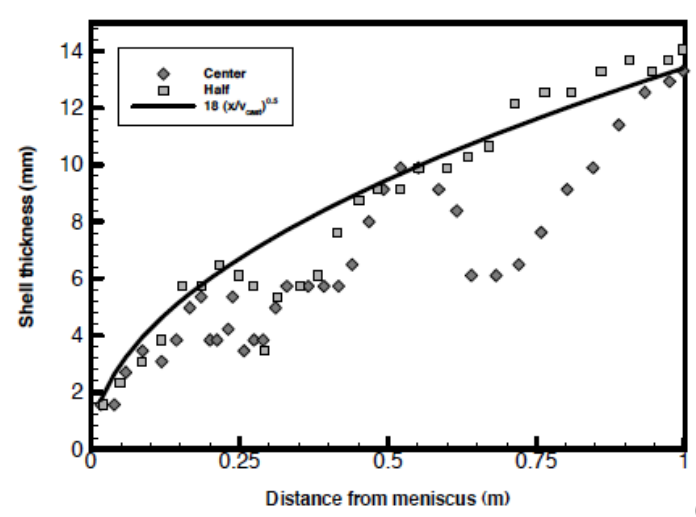

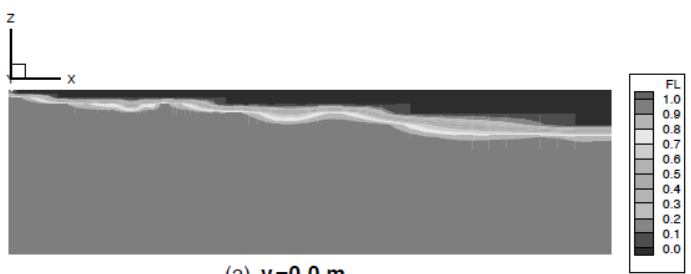

(a) $\mathbf{y}=0.0 \mathrm{~m}$

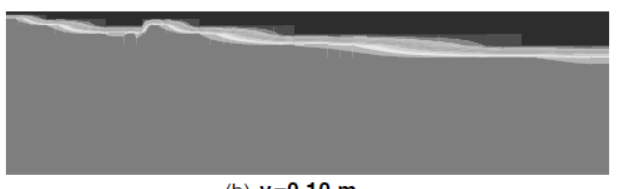

(b) $\mathbf{y}=0.10 \mathrm{~m}$ (b),

Figura 3. (a) Crescimento da espessura de casca calcula pelo modelo e pela equação $18(x / v)^{0,5}$; (b) Mapas de fração líquida em duas posições ao longo da placa [5].

Já Rodrigo Ottoni da Silva Pereira [6] propôs, em sua tese de doutorado, um modelo matemático para o lingotamento contínuo de placas, incluindo fenômenos de escoamento e solidificação. Um dos resultados obtidos por Pereira [6], ilustrados pela Figura 4, foi a comparação da espessura de casca calculada por diferentes modelos matemáticos onde: "CS" é a camada solidificada; "CP" a camada pastosa; "fluxo" é o modelo acoplado com escoamento, transferência de calor e solidificação; "term" é o modelo sem escoamento e "segreg" é o modelo acoplado com segregação. É interessante notar a influência que os modelos possuem no perfil da isoterma liquidus (CP). Esse perfil é para a face larga da placa. Na face estreita as diferenças entre os modelos tendem a ser mais pronunciadas. O modelo com segregação indica um princípio mais tardio da solidificação, levando a uma região

* Contribuição técnica ao $45^{\circ}$ Seminário de Aciaria - Internacional, 25 a 28 de maio de 2014, 


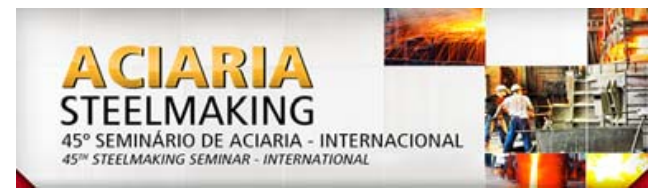

pastosa mais estreita. Observa-se também que o modelo sem escoamento (term) tende a apresentar diferenças com os modelos envolvendo o fluxo.

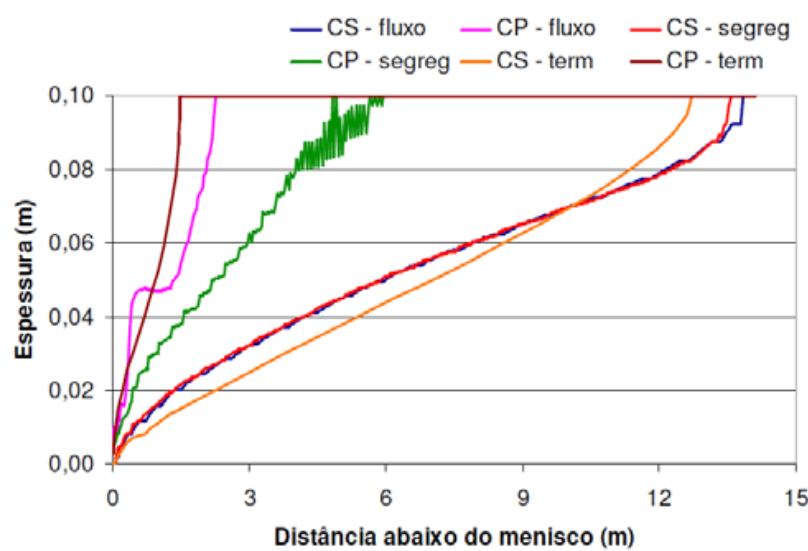

Figura 4. Comparação da espessura das camadas solidificada (CS) e pastosa (CP) perpendicular à face larga para os diferentes modelos matemáticos [6].

Como pode ser observado, todos os trabalhos listados anteriormente possuem como foco principal, a estimativa da espessura de casca e o comprimento metalúrgico quando considerados os fenômenos de escoamento, transferência de calor e solidificação. Entretanto, em nenhum deles foi mencionado o uso de agitadores eletromagnéticos, seja para tarugos de seção quadrada ou redonda. E, como já foi referido por Kunstreich [2], os efeitos desses equipamentos na estrutura solidificada são de suma importância.

\section{METODOLOGIA}

\subsection{Modelagem Fluidodinâmica do Escoamento e Solidificação no Molde do Lingotamento Contínuo sob o Efeito de Agitação Eletromagnética}

O objetivo desta etapa foi determinar o efeito da agitação eletromagnética na remoção do calor de superheat no interior do molde. Para isso foi utilizado um modelo em CFD (Computational Fluid Dynamics) capaz de acoplar os efeitos da agitação eletromagnética ao escoamento do aço e a solidificação no interior do molde, determinando a distribuição de temperaturas e a fração líquida ao longo da seção do tarugo, na saída do molde. O software adotado foi o Ansys/Fluent para a solução das RANS (Reynolds Average Navier-Stokes equations) [7], com transferência de calor e solidificação, incluindo ainda as forças de Lorentz para as diferentes condições de agitação simuladas. Detalhes do modelo eletromagnético podem ser encontrados em Barroso et al. [1] .

As equações que regem os fenômenos de escoamento e transferência de calor com solidificação estão descritas a seguir (Equações 1 a 8). De forma simultânea à solução do escoamento, a equação de balanço de energia (entalpia) é resolvida, onde no termo fonte $S$ é inserida a liberação de calor latente que ocorre durante a mudança de fase entre liquidus e solidus.

$$
\frac{\partial}{\partial t}(\rho H)+\nabla \cdot(\rho \vec{U} H)=\nabla \cdot(k \nabla T)+S
$$

onde $H=h+\Delta H$ e $h=C_{p} \cdot T$. A liberação de calor latente pode ser expressa em termos do calor latente de solidificação $(L)$ e a fração líquida $(\beta)$ :

* Contribuição técnica ao $45^{\circ}$ Seminário de Aciaria - Internacional, 25 a 28 de maio de 2014, 


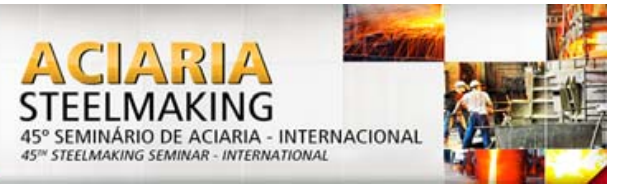

$$
\Delta H=\beta L
$$

A fração líquida $(\beta)$ é definida como:

$$
\begin{aligned}
& \beta=0 \text { para } T \leq T_{\text {solidus }} \quad \beta=1 \text { para } T \geq T_{\text {liquidus }} \\
& \qquad \beta=\frac{T-T_{\text {solidus }}}{T_{\text {liquidus }}-T_{\text {solidus }}} \text { para } T_{\text {solidus }}<T<T_{\text {liquidus }}
\end{aligned}
$$

Concomitantemente à solução da equação de energia, resolvem-se as equações de escoamento e do modelo de turbulência, neste caso optado pelo tradicional $k-$ epsilon. As equações da continuidade, balanço de momentum e do modelo de turbulência estão descritas abaixo.

$$
\begin{gathered}
\frac{\partial \rho}{\partial t}+\nabla \cdot \rho \vec{U}=0 \\
\frac{\partial}{\partial t}(\rho \vec{U})+\nabla \cdot(\rho \vec{U} \otimes \vec{U})=\vec{B}-\nabla p+\nabla \cdot\left(\mu_{e f f}\left(\nabla \vec{U}+(\nabla \vec{U})^{T}\right)\right) \\
\frac{\partial}{\partial t}(\rho k)+\rho \nabla \cdot(\vec{U} k)=\nabla \cdot\left(\mu_{e f f} / \sigma_{k} \nabla k\right)+P_{k}-\rho \varepsilon \\
\frac{\partial}{\partial t}(\rho \varepsilon)+\rho \nabla \cdot(\vec{U} \varepsilon)=\nabla \cdot\left(\mu_{e f f} / \sigma_{\varepsilon} \nabla \varepsilon\right)+\frac{\varepsilon}{k}\left(c_{\varepsilon 1} P_{k}-c_{\varepsilon 2} \rho \varepsilon\right)
\end{gathered}
$$

Na Mushy Zone, ou região pastosa, a fração líquida varia entre 0 e 1. Essa zona é modelada como um "pseudo" meio poroso, onde a porosidade diminui de 1 para 0 à medida que o material solidifica, originando um aumento da viscosidade e da densidade, reduzindo as velocidades. Para incluir este efeito na região da casca, um termo fonte para a equação de momentum é considerado:

$$
S=\frac{(1-\beta)^{2}}{\beta^{2}+\delta} A_{m u s h}\left(\vec{U}-\vec{V}_{L}\right)
$$

sendo $\vec{V}_{L}$ a velocidade de lingotamento, $A_{\text {mush }}$ e $\delta$ constantes. Um termo similar é aplicado para as equações de turbulência.

\subsection{Geometria e Condições de Contorno}

A malha computacional, que compreende o domínio do molde com comprimento de $0,8 \mathrm{~m}$, é apresentada na Figura 5 . A temperatura de entrada do aço é dada pela temperatura liquidus deste tipo de aço acrescida da temperatura de superheat. $\mathrm{O}$ topo do molde foi considerado adiabático e as paredes assumindo um coeficiente de transferência de calor em função da velocidade de lingotamento, conforme Tabela 1.

* Contribuição técnica ao 45 Seminário de Aciaria - Internacional, 25 a 28 de maio de 2014, Porto Alegre, RS, Brasil. 


\section{ACIARIA}
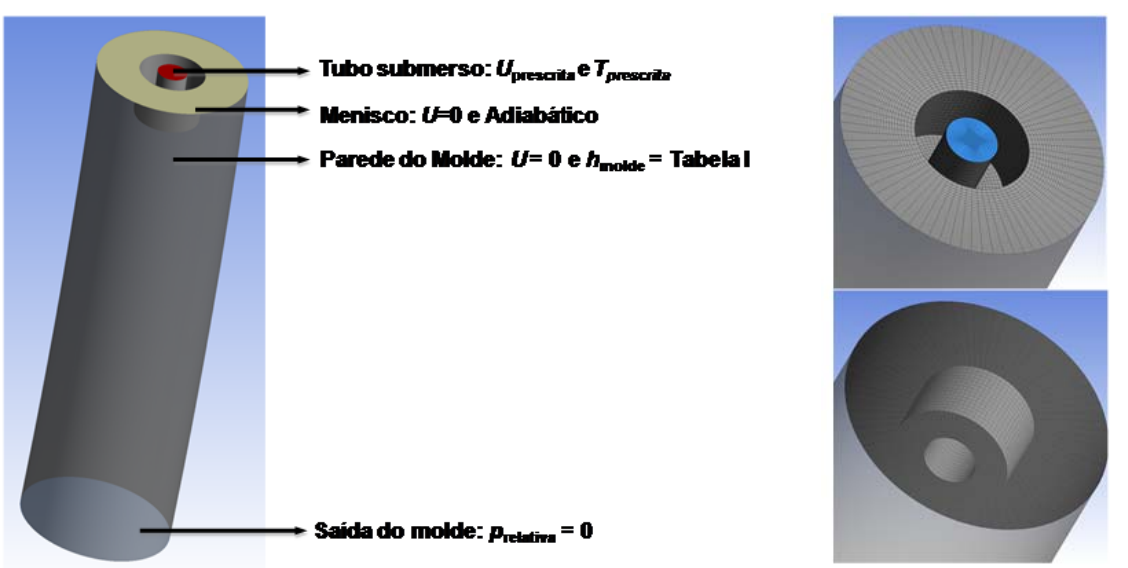

(a)

(b)

Figura 5. (a) Geometria simulada e condições de contorno; e (b) Detalhe da geometria e malha na saída da válvula submersa.

Tabela 1. Coeficiente de transferência de calor no molde em função da velocidade de lingotamento

\begin{tabular}{|c|c|}
\hline $\begin{array}{c}\text { Velocidade de lingotamento }-\mathbf{V}_{\mathbf{L}} \\
{[\mathrm{m} / \mathbf{m i n}]}\end{array}$ & $\begin{array}{c}\text { Coeficiente de transferência de } \\
\text { calor no } \mathbf{m o l d e} \boldsymbol{h}_{\text {molde }}\left[\mathbf{W} / \mathbf{m}^{2} . \mathbf{K}\right]\end{array}$ \\
\hline 0,8 & 1465 \\
\hline 1,0 & 1641 \\
\hline 1,2 & 1798 \\
\hline 1,4 & 1940 \\
\hline 1,6 & 2070 \\
\hline 1,8 & 2198 \\
\hline
\end{tabular}

$\mathrm{Na}$ Tabela 2 estão descritos os parâmetros de processo e as condições operacionais empregadas nas simulações do modelo.

Tabela 2. Parâmetros e condições operacionais empregadas

\begin{tabular}{|l|l|l|l|}
\hline Variável & Valor & Variável & Valor \\
\hline Diâmetro do molde & $230 \mathrm{~mm}$ & Temperatura liquidus & $1520^{\circ} \mathrm{C}$ \\
\hline $\begin{array}{l}\text { Comprimento do } \\
\text { molde }\end{array}$ & $800 \mathrm{~mm}$ & Temperatura solidus & $1470^{\circ} \mathrm{C}$ \\
\hline $\begin{array}{l}\text { Diâmetro interno } \\
\text { da válvula }\end{array}$ & $45 \mathrm{~mm}$ & $\begin{array}{l}\text { Calor latente de solidificação } \\
(\mathrm{L})\end{array}$ & $203.000 \mathrm{~J} / \mathrm{kg}$ \\
\hline $\begin{array}{l}\text { Submersão da } \\
\text { válvula }\end{array}$ & $65 \mathrm{~mm}$ & Densidade do aço $(\rho)$ & $8.000 \mathrm{~T}-0,64 \mathrm{~T}$ \\
\hline $\begin{array}{l}\text { Correntes de } \\
\text { agitação }(\mathrm{M}-\mathrm{EMS})\end{array}$ & $0,61,100,183,350 \mathrm{~A}$ & Viscosidade do aço líquido $(\mu)$ & $0,00553 \mathrm{~kg} / \mathrm{m} . \mathrm{s}$ \\
\hline $\begin{array}{l}\text { Temperatura de } \\
\text { lingotamento }\end{array}$ & $1553^{\circ} \mathrm{C}$ & $\begin{array}{l}\text { Condutividade térmica do aço } \\
\text { líquido }(k)\end{array}$ & $30 \mathrm{~W} / \mathrm{m} . \mathrm{K}$ \\
\hline $\begin{array}{l}\text { Temperatura de } \\
\text { superheat }\end{array}$ & $33^{\circ} \mathrm{C}$ & $\begin{array}{l}\text { Calor específico do aço líquido } \\
\left(C_{P}\right)\end{array}$ & $680 \mathrm{~J} / \mathrm{kg} \cdot \mathrm{K}$ \\
\hline
\end{tabular}

* Contribuição técnica ao 45 Seminário de Aciaria - Internacional, 25 a 28 de maio de 2014, Porto Alegre, RS, Brasil. 


\section{RESULTADOS E DISCUSSÃO}

As simulações mostraram que a espessura da casca na saída do molde varia em função da velocidade de lingotamento, conforme ilustrado pela Figura 6 abaixo. A Figura 6 mostra o mapa de escoamento e a casca solidificada junto às paredes para duas velocidades de lingotamento: $1,0 \mathrm{~m} / \mathrm{min}$ (esquerda) e 1,8 m/min (direita). Note que a espessura da casca na saída do molde é maior para velocidades mais baixas.

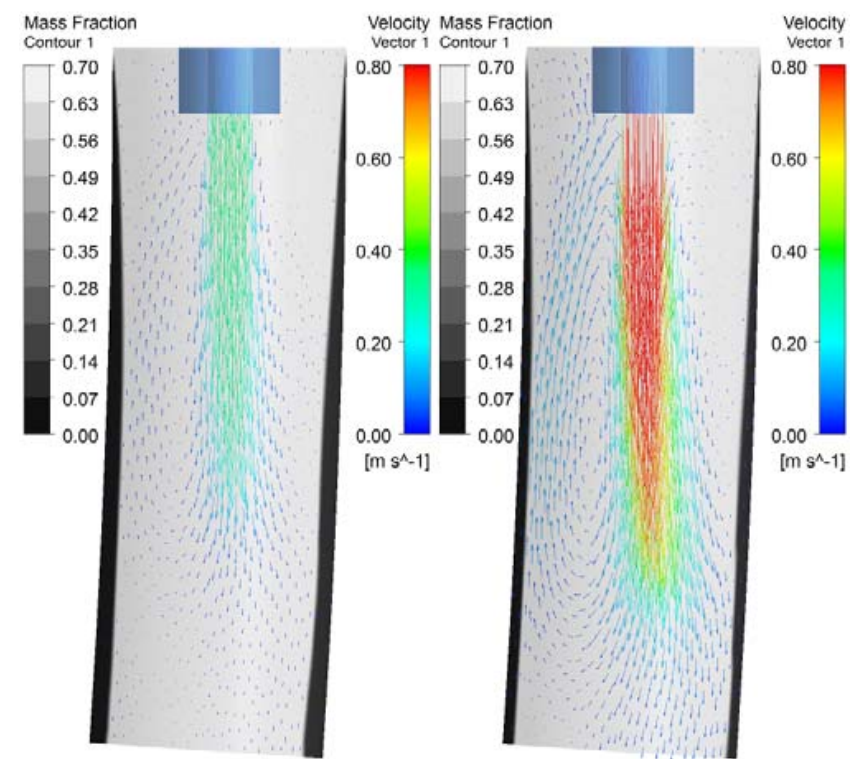

Figura 6. Mapas de velocidade e fração líquida para duas velocidades de lingotamento.

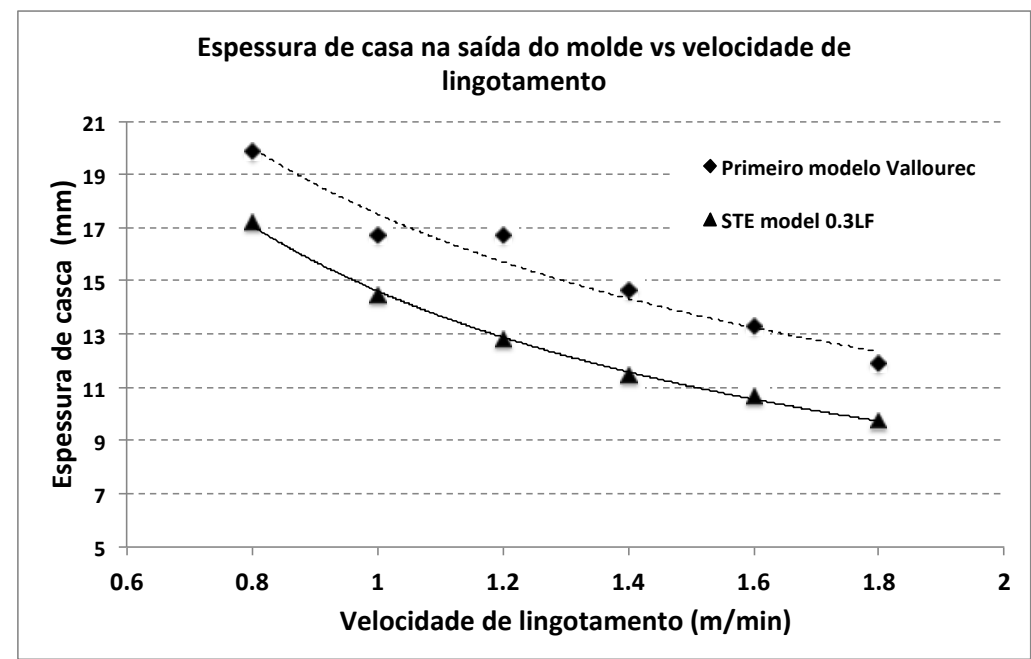

Figura 7. Efeito do aumento da velocidade de lingotamento na espessura de casca.

Este mesmo efeito pode ser observado na Figura 7 que apresenta os valores médios de espessura de casca para cada velocidade de lingotamento e os compara com os resultados obtidos pelo primeiro modelo da Vallourec [10]. Esse comportamento já era esperado, pois quanto mais elevada for a velocidade de lingotamento, maior é o coeficiente de transferência de calor no molde $h_{\text {molde }}$ (Tabela 1 ). O valor de $h_{\text {molde }}$ é calculado a partir da diferença de temperatura da água de refrigeração do molde $(\Delta \mathrm{T})$. Como a vazão de água de refrigeração é mantida praticamente constante, a diferença de temperatura tende a aumentar e, consequentemente, elevar também o

* Contribuição técnica ao $45^{\circ}$ Seminário de Aciaria - Internacional, 25 a 28 de maio de 2014, 
valor de $h_{\text {molde. }}$ Com relação à determinação da espessura da casca, não há uma regra bem definida, porém, segundo Aboutalebi et al.[3], é mais comum que ela seja obtida quando a fração liquida for menor que 30\%. A diferença média observada entre os modelos, que é da ordem de $2,8 \mathrm{~mm}$, pode estar associada ao fato de que o primeiro modelo não simula o escoamento de forma concomitante à transferência de calor e a solidificação, pois se trata de um modelo de condução de calor.

Uma vez conhecida a espessura da casca em função da velocidade de lingotamento, o próximo passo foi avaliar se o agitador eletromagnético do molde (M-EMS) também pode influenciar, de alguma forma, nessa espessura e no perfil térmico.

O comportamento da espessura da casca em função da corrente do M-EMS é ilustrado pela Figura $8 a$ a seguir. Observe que há um aumento da espessura com a corrente, porém ele tende a se estabilizar para correntes mais elevadas. O agitador, por causa dos efeitos de convecção, auxilia na extração de calor do molde, implicando num aumento da espessura da casca e na redução do comprimento metalúrgico. Este "ajuste da condutividade térmica", foi estudado por Karlinski [11] que avaliou um parâmetro, chamado "condutividade térmica efetiva", sobre as isotermas liquidus e solidus, que busca incluir no modelo de solidificação desenvolvido, os efeitos da agitação eletromagnética. Em sua tese de doutorado, o autor variou o valor de $k$ original e pode perceber que o modelo é sensível a essa variação. Porém, admite que esse número seja difícil de ser estimado e precisa ser ajustado a partir de medições de temperatura feitas no molde.
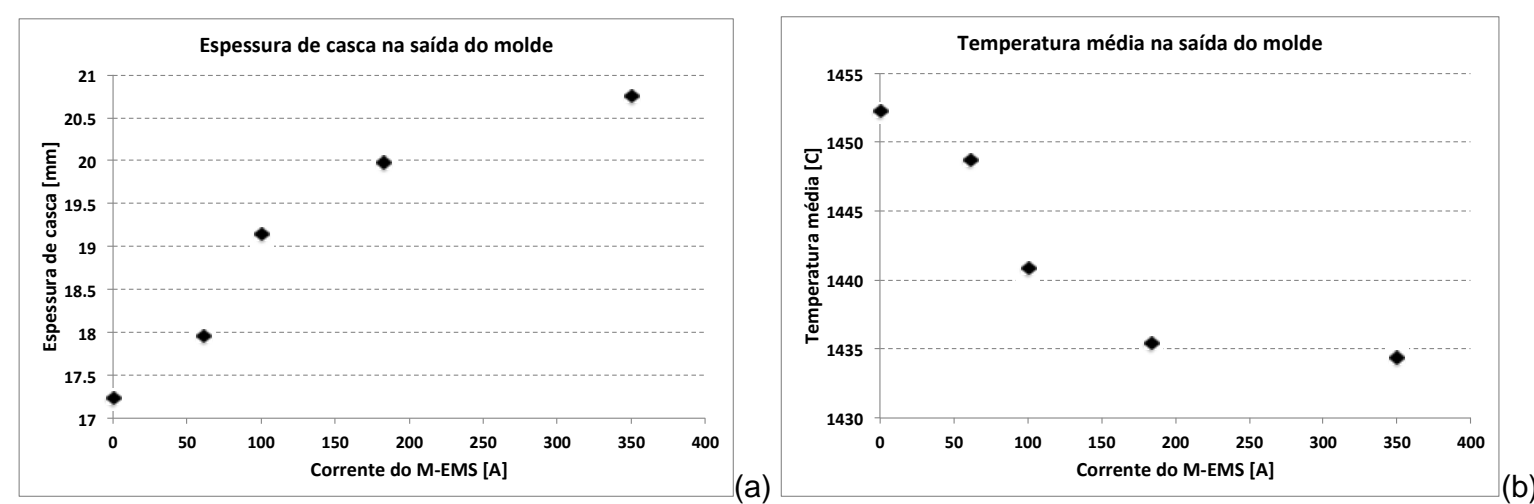

Figura 8. (a) Espessura de casca na saída do molde; e (b) Temperatura média na saída do molde em função da corrente do agitador do molde.

Essa "casca mais espessa" na saída do molde pode ser considerada reflexo da queda do valor médio de temperatura da barra nesta situação, conforme ilustrado pela Figura 8b. Note que, com o aumento da agitação eletromagnética, o valor médio da temperatura apresenta um comportamento de queda, com tendências a se estabilizar para correntes em torno de 200A.

Essas constatações são ratificadas pela Figura 9, a seguir, que simulou, para duas condições extremas, $0 \mathrm{~A}$ e $350 \mathrm{~A}$, as linhas de corrente dentro do molde. Observe que elas são completamente diferentes bem como os valores de velocidade junto à casca solidificada. Sem agitação, o movimento do aço ocorre basicamente na vertical, enquanto para valores de corrente de agitação mais elevados, predomina a movimentação rotacional que atinge velocidades lineares de $0,46 \mathrm{~m} / \mathrm{s}$. É importante ressaltar que, neste comparativo, a velocidade de lingotamento foi mantida fixa em $0,8 \mathrm{~m} / \mathrm{min}$ bem como os valores de coeficiente de transferência de calor no molde.

* Contribuição técnica ao 45 Seminário de Aciaria - Internacional, 25 a 28 de maio de 2014, Porto Alegre, RS, Brasil. 

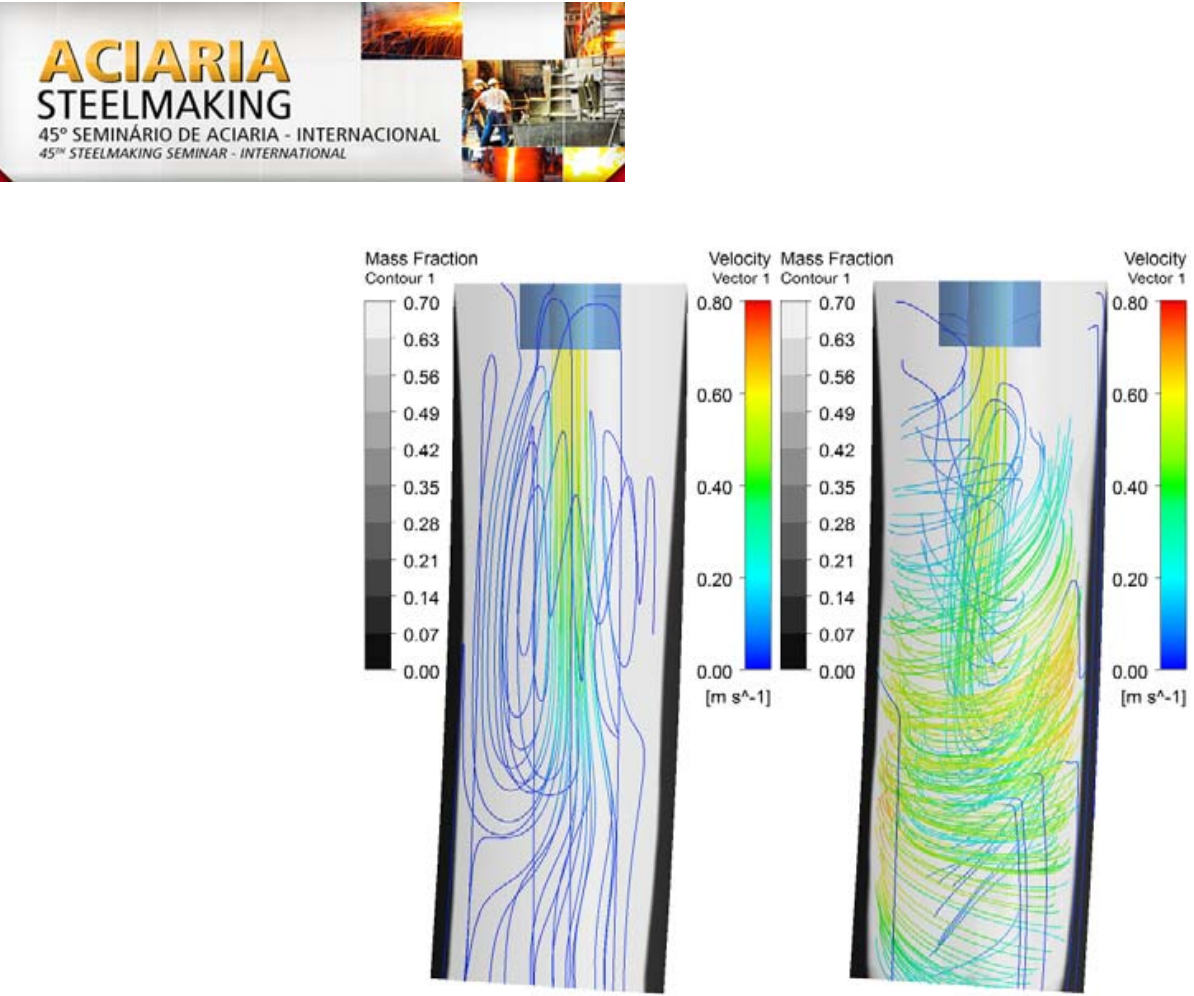

Figura 9. Mapas de velocidade e fração líquida com o M-EMS desligado (0A) e com 350A.

Os resultados, em termos de solidificação, são apresentados na Figura 10 que mostra o perfil de fração líquida ao longo da seção na saída do molde. Quando não se agita, praticamente não é observada a formação de sólidos na região central. A fração líquida é quase 100\%. Entretanto, este valor pode estar superestimado, uma vez que, na simulação, se assume um contato perfeito entre o aço líquido e as paredes do molde e os efeitos relativos ao pó fluxante e oscilação do molde também são desconsiderados.

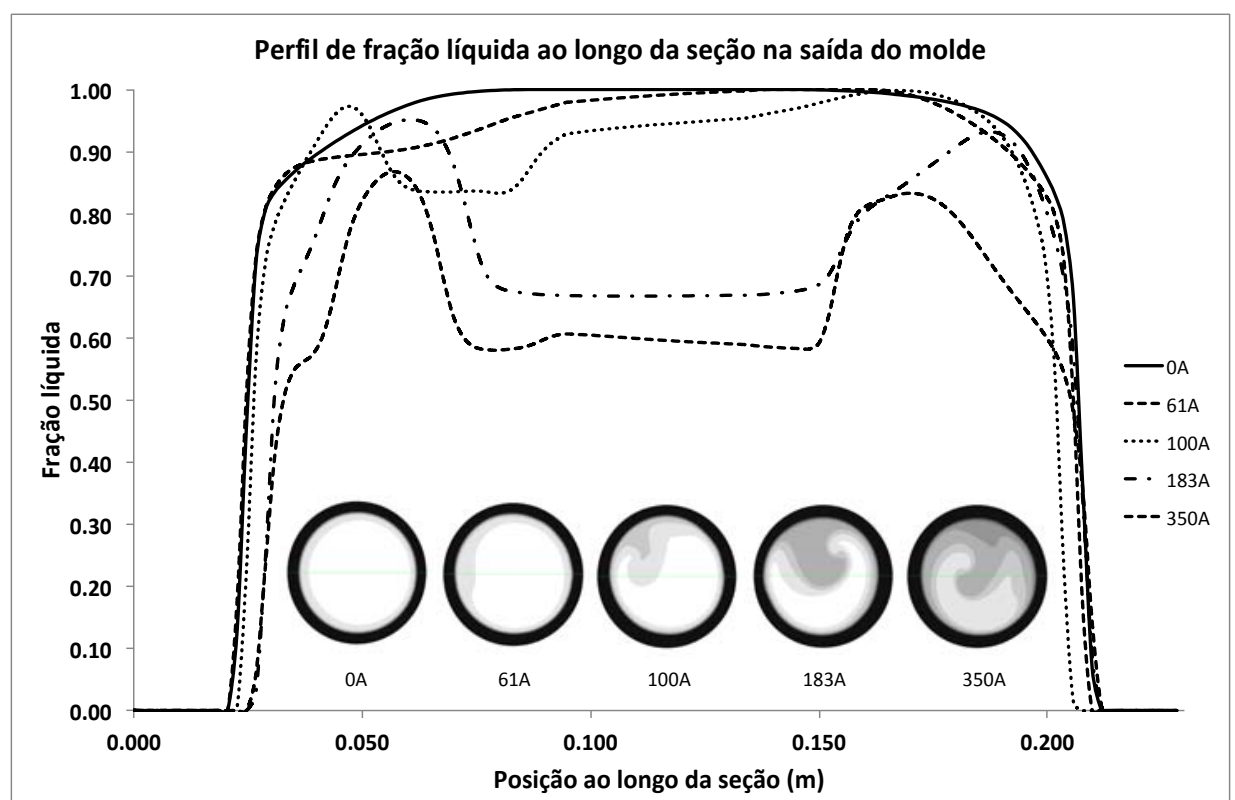

Figura 10. Perfil de fração líquida na saída do molde em função da corrente do agitador M-EMS.

À medida que a corrente de agitação eletromagnética aumenta, há uma redução na fração líquida no centro da seção ("miolo da barra"), chegando a valores de fração sólida de até 40\% (para 350A). Essa condição é mais condizente com a realidade, uma vez que a agitação ajuda a reduzir o superheat e aumentar a região equiaxial de solidificação, conforme já mostrado por Barroso et al. [1]. Naquela oportunidade o

* Contribuição técnica ao $45^{\circ}$ Seminário de Aciaria - Internacional, 25 a 28 de maio de 2014, Porto Alegre, RS, Brasil. 


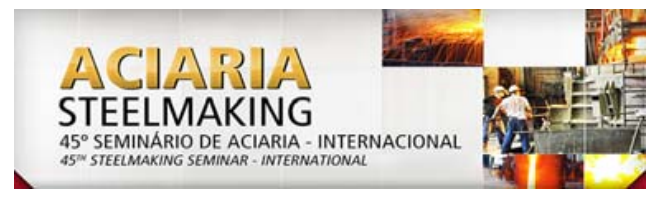

autor demonstrou que a zona equiaxial cresceu de $5 \%$ para $14 \%$ com a corrente variando de $60 \mathrm{~A}$ para 180A. Observando as figuras $8 \mathrm{~b}$ e 10, nessa mesma situação, a simulação indicou uma queda na temperatura média do tarugo de aproximadamente $13^{\circ} \mathrm{C}$ e um aumento da fração sólida em torno de $30 \%$.

Os perfis de fração líquida, ilustrado esquematicamente pela Figura 10 (quanto mais claro mais fração líquida), podem variar ainda em função do modelo de determinação da fração sólida. Neste estudo foi usada a regra da alavanca, com o coeficiente de partição constante, e assumindo uma variação linear da fração sólida com a temperatura, segundo a Equação 3. Porém, como comenta Karlinski [11], a variação da fração sólida tende a ser parabólica com a temperatura, como mostra a Figura 11, indicando que pode haver mais fração líquida em temperaturas mais próximas da sólida.

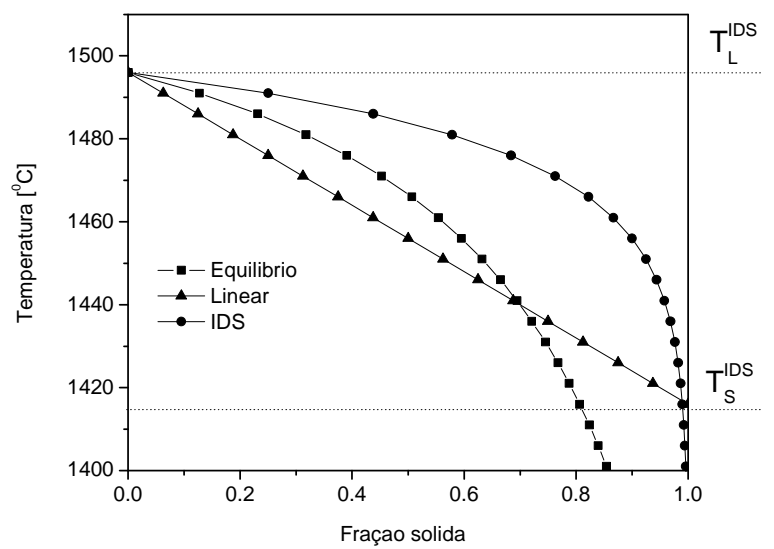

Figura 11. Evolução da fração sólida para diferentes equações de fs com as temperaturas solidus e liquidus calculadas com o modelo IDS.

Cabe ainda ressaltar a forma não simétrica das curvas com agitação eletromagnética mostrada esquematicamente na figura 10. A explicação para este fato está no escoamento não uniforme decorrente da posição excêntrica do molde com relação ao agitador que pode ser verificada na Figura 12. A distância das bobinas (destacadas pelos quadrados pontilhados) em relação ao eixo central do molde não é a mesma.

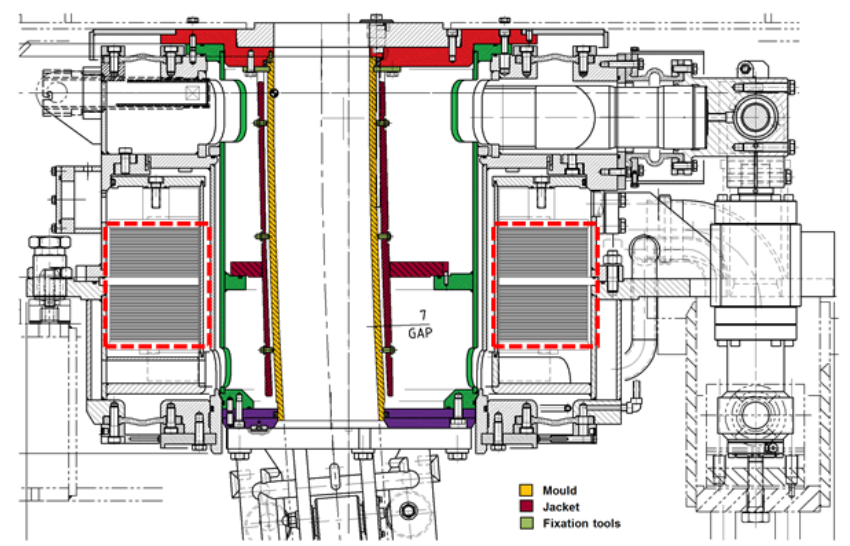

Figura 12. Posição excêntrica do molde em relação às bobinas do agitador M-EMS.

Os resultados aqui apresentados são preliminares e uma investigação mais detalhada se faz necessária para verificar as previsões do modelo. Como já foi

* Contribuição técnica ao 450 Seminário de Aciaria - Internacional, 25 a 28 de maio de 2014, Porto Alegre, RS, Brasil. 
comentado, alguns parâmetros de modelagem e condições de contorno empregadas precisam ser melhor avaliados, a saber:

- Evolução da fração sólida com a temperatura. Testar diferentes modelos que considerem a composição química do aço, principalmente em aços ligados;

- Verificar o coeficiente de transferência de calor no molde sob diferentes correntes de agitação. Essa análise pode ser feita através de uma instrumentação do molde com termopares, controle preciso da temperatura da água do molde ou modelagem do molde em si.

\section{CONCLUSÕES}

Os resultados mostraram que as tendências observadas pelas simulações estão dentro do esperado e de acordo com a literatura.

A agitação eletromagnética realmente promove uma mudança nos perfis de fração liquida na saída do molde, aumentando a região de fração sólida no centro do tarugo. Esse efeito pode estar relacionado à formação da zona equiaxial de solidificação.

O aumento da corrente do agitador contribui para a elevação da espessura da casca solidificada. Sua comprovação prática não é trivial, pois seria necessário ocorrer um rompimento do veio e, com sorte a coleta o material remanescente para que se pudesse medir a parede solidificada. Ou então, tal efeito poderia ser checado através das marcas de bandas brancas, provocadas pelo agitador na zona final de solidificação, o que não ocorreu durante o experimento.

O aumento da corrente do agitador também contribuiu para a redução da temperatura média na saída do molde, segundo as simulações. Esse resultado é consequência do aumento da extração de calor em decorrência da agitação eletromagnética.

Por fim, é importante ressaltar que apesar da coerência com os testes experimentais realizados no trabalho de Barroso et al. [1] e a bibliografia, é recomendado que sejam conduzidos estudos mais aprofundados, no que tange a avaliação da evolução da fração sólida com a temperatura em função da composição química do aço e/ou a verificação do coeficiente de transferência de calor no molde sob diferentes correntes de agitação, que pode ser feita através de uma instrumentação do molde com termopares, controle preciso da temperatura da água do molde ou modelagem do molde em si.

\section{REFERÊNCIAS}

1 Barroso R, Gandra AP, Miranda MC, Nadalon JEA, Trindade LB, Contini AC. Estudo da influência do uso de agitadores eletromagnéticos na remoção do calor de superheat no lingotamento contínuo de aços. In: 44 Seminário de Aciaria Internacional; maio 2013; Araxá, Brasil. São Paulo: ABM; 2013. p.519-529.

2 Kunstreich S. Electromagnetic stirring for continuous casting - Part 2. La Revue de Métallurgie - CIT. 2003; Nov.:1043-61.

3 Aboutalebi MR, Hasan ME, Guthrie RIL. Coupled turbulent flow, heat, and solute transport in continuous casting processes. Metallurgical and Materials Transactions B. 1995;26b:731-744.

4 Seyedein $\mathrm{SH}$, Hasan M. A three-dimensional simulation of coupled turbulent flow and macroscopic solidification heat transfer for continuous slab casters. Int. J. Mass Transfer. 1997;40(9):4405-23.

\footnotetext{
* Contribuição técnica ao 45 Seminário de Aciaria - Internacional, 25 a 28 de maio de 2014, 
5 Ha MY, Lee HG, Seong S H. Numerical simulation of three-dimensional flow, heat transfer and solidification of steel in continuous casting mold with electromagnetic brake. Journal of Materials Processing Technology. 2003;133:322-39.

6 Pereira ROS. Modelamento matemático do escoamento turbulento, da transferência de calor e da solidificação no distribuidor e na máquina de lingotamento contínuo [tese de doutorado]. Belo Horizonte: Universidade Federal de Minas Gerais; 2004.

7 Ansys/Fluent. Theory Guide. Release 14.5.

8 Ayata K, Mori T, Fujimoto T, Ohnishi T, Wakasugi I. Improvement of Macrosegregation in Continuously Cast Bloom and Billet by Electromagnetic Stirring. Transactions ISIJ. 1984;24:931-939.

9 Szekeres ES. Brimacombe continuous casting course - Strand solidification and related imperfections [course]; May 2010. Item N, p.17-18. Vancouver: Brimacombe Continuous Casting Society; 2010.

10 Prevost B, Ladeuille L, Passagli D, Bellet M. Relatório Técnico do Grupo Vallourec; Project of Parabolic Mold. Barreiro: Vallourec; 2007. p.78.

11 Karlinski V. Desenvolvimento de software de simulação da solidificação de aços no processo de lingotamento contínuo de tarugos [tese de doutorado]. Porto Alegre: Universidade Federal do Rio Grande do Sul; 2011.

* Contribuição técnica ao 45 Seminário de Aciaria - Internacional, 25 a 28 de maio de 2014 , 